\title{
Space- and time-resolved diagnostics of the ENEA EUV discharge-produced-plasma source used for metrology and other applications
}

\author{
S. Bollanti, P. Di Lazzaro, F. Flora, L. Mezi, D. Murra, and A. Torre \\ ENEA, FSN-Fusion Physics Division-Radiation Sources, Antennas, and Diagnostics Laboratory, Frascati, Italy \\ (Received 10 May 2015; revised 14 September 2015; accepted 30 October 2015)
}

\begin{abstract}
A discharge-produced-plasma (DPP) source emitting in the extreme ultraviolet (EUV) spectral region is running at the ENEA Frascati Research Centre. The plasma is generated in low-pressure xenon gas and efficiently emits 100-ns duration radiation pulses in the 10-20-nm wavelength range, with an energy of $20 \mathrm{~mJ} / \mathrm{shot} / \mathrm{sr}$ at a $10-\mathrm{Hz}$ repetition rate. The complex discharge evolution is constantly examined and controlled with electrical measurements, while a nsgated CCD camera allowed observation of the discharge development in the visible, detection of time-resolved plasmacolumn pinching, and optimization of the pre-ionization timing. Accurately calibrated Zr-filtered PIN diodes are used to monitor the temporal behaviour and energy emission of the EUV pulses, while the calibration of a dosimetric film allows quantitative imaging of the emitted radiation. This comprehensive plasma diagnostics has demonstrated its effectiveness in suitably adjusting the source configuration for several applications, such as exposures of photonic materials and innovative photoresists.
\end{abstract}

Keywords: discharge produced plasma; EUV; extreme ultraviolet; plasma diagnostics; xenon plasma

\section{Introduction}

For many years, low-temperature plasmas emitting extreme ultraviolet (EUV) radiation (photon energy: $h v=$ 20-284 eV, wavelength: $\lambda=4.4-62 \mathrm{~nm}$ ) have been the object of strong interest and intense development and study by both industry and academia. In fact, EUV radiation, thanks to its short wavelength, its short penetration length in matter (typically a few tens of $\mathrm{nm}$ ), and the availability of high-reflectivity $(\geqslant 70 \%$ at $\lambda=13-15 \mathrm{~nm})$ normal-incidence multilayer mirrors, allows patterning at a high spatial resolution, for example, on photoresists and on photonic materials. This led to the development of high-averagepower plasma sources for EUV-radiation-based lithography systems for microelectronics, and also to low- to mediumpower sources for metrology and tests on mirrors, innovative materials etc. Basically, two kinds of EUV plasma sources have been developed, laser-produced plasmas (LPPs) and discharge-produced plasmas (DPPs). For an extensive review on plasma sources for EUV lithography, see Ref. [1] and references therein. DPPs have been investigated using a number of configurations, such as capillary, Z-pinch, and

Correspondence to: S. Bollanti, ENEA FRASCATI Research Centre, via Enrico Fermi 45, 00044 Frascati (Rome), Italy.

Email: sarah.bollanti@enea.it plasma focus, under various operating conditions, e.g., using different gases, geometries and time scales ${ }^{[2-5]}$.

An EUV DPP source is currently operating at the ENEA Frascati Research Centre. It was originally realized within a collaboration between ENEA and the Physics Department of the University of L'Aquila in the frame of the National FIRBEUVL Project ${ }^{[6]}$. The source has successively undergone appropriate modifications and upgrades at ENEA in order to greatly improve its performance ${ }^{[7]}$. After a brief description of its operational principles, we will report on source characterization by means of various diagnostic tools, which can be applied as well to LPPs. Finally, some source applications involving the treatment of innovative photoresists and photonic materials will be illustrated.

\section{EUV plasma source description}

A picture of the ENEA DPP source and the related scheme are reported in Figure 1, while in Figure 2 a simplified electrical pumping scheme is depicted.

A low-inductance 50-nF glycol cylindrical capacitor is charged to $V_{1} \approx 18-20 \mathrm{kV}$ and then rapidly discharged through a spark-gap switch and a thin-metallic-tube electrode in low-pressure (0.5-1.0 mbar) Xe gas, which fills 

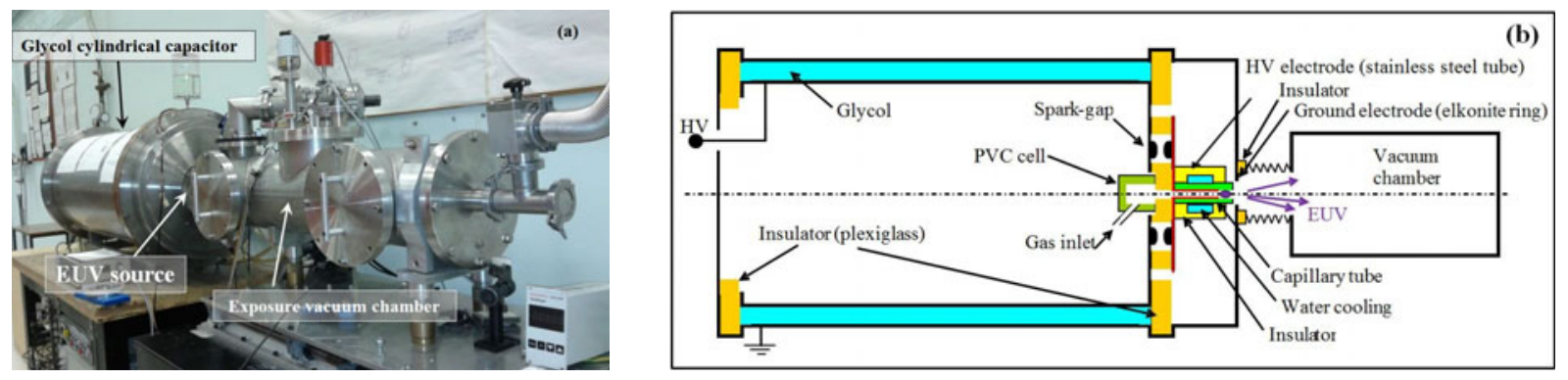

Figure 1. ENEA DPP source: (a) picture; (b) schematic.

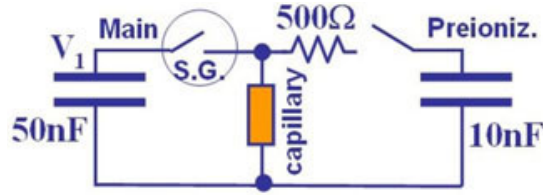

Figure 2. Simplified electrical scheme of the DPP pumping circuit.

a short alumina capillary tube, towards the ground annular electrode. This produces the main discharge (11-kA peak current, 240-ns half-period), which follows the lowcurrent (20-30 A, almost constant) long-duration $(\approx 2 \mu \mathrm{s})$ pre-ionization phase, and leads to high-temperature plasma formation and pinching towards the capillary axis thanks to the resulting magnetic field $(>1 \mathrm{~T}$ at the capillary surface). Consequently, the plasma resistance rises and the temperature increases up to $30-40 \mathrm{eV}$, where the hot plasma emits radiation peaked in the EUV region before relaxation and cooling. In typical operating conditions, the DPP source emits EUV pulses having an energy of $20 \mathrm{~mJ} / \mathrm{shot} / \mathrm{sr}$ in the $\lambda=10-20 \mathrm{~nm}$ range, with a pulse duration of $100 \mathrm{~ns}$. The temporal behaviour of the high voltage on the glycol capacitor, as detected by using an appropriate calibrated resistive divider, is shown in Figure 3, together with the main discharge current measured with a current pulse measurement transformer (EEV, mod. MA459B), and the emitted EUV radiation pulse detected by a fast PIN diode (IRD, mod. AXUV20HS1BNC) filtered by a 150-nm-thick $\mathrm{Zr}$ foil to select the $\lambda=10-20 \mathrm{~nm}$ wavelength range ${ }^{[8]}$. The PIN diode, powered in DC mode, is connected to the oscilloscope through a decoupling capacitor which slightly modifies the signal and generates a small negative noise after each falling edge; this can be observed for example at 0.22 and $0.45 \mu \mathrm{s}$. A correction of this effect has been omitted because of its small magnitude. The EUV emission occurs after the plasma compression, in all the first three current oscillations, in which the lower the current peak, the longer the time required to reach the compression. At a repetition rate of $10 \mathrm{~Hz}$ the rms pulse-to-pulse stability is $4 \%$.

The minimum transverse dimension of the EUV source is $0.3 \mu \mathrm{m}$, measured by a $\mathrm{Zr}$-filtered $100-\mu \mathrm{m}$-diameter pinhole

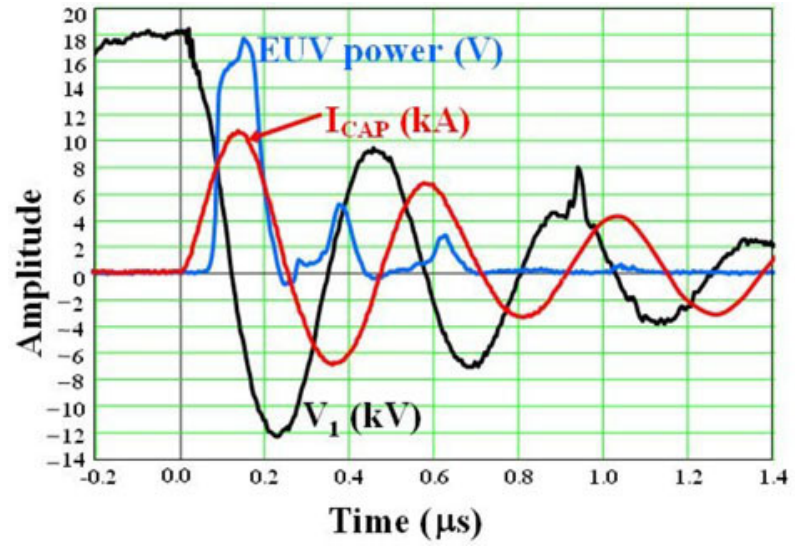

Figure 3. Temporal evolution of the glycol capacitor voltage $V_{1}$, the main discharge current $I_{\mathrm{CAP}}$ and the EUV signal (see text for details on the related diagnostics).

camera and a Gafchromic dosimetric film. Ionic debris are considerably deflected by a dipole magnet, allowing the exploitation of clean radiation. An extensive description of the DPP source can be found in Ref. [7].

\section{Absolute calibration of PIN diodes}

When using a plasma source to carry out controlled irradiation, an accurate evaluation of the released fluences (i.e., energy per unit area) in a given spectral interval is needed. The IRD AXUV series photodiodes have near-theoretical quantum efficiencies for EUV photons, which can be predicted by the expression $h v / 3.65$, where $h v$ is the photon energy in $\mathrm{eV}^{[9]}$. These detectors thus allow full control of the source irradiance during sample exposure. In particular, to this end we used two high-speed absolute PIN diodes (IRD, mod. AXUV20HS1) placed in the vacuum chamber (see Figure 1(a)): the measurement diode is mounted off axis (with respect to the plasma source geometry), is filtered by a 300-nm-thick, Ni-mesh-supported Zr foil (Luxel Corp.), and is kept fixed in that position during the exposures; the other, reference diode, is on axis and is initially used to calibrate the measurement diode, since the exposed samples are normally 


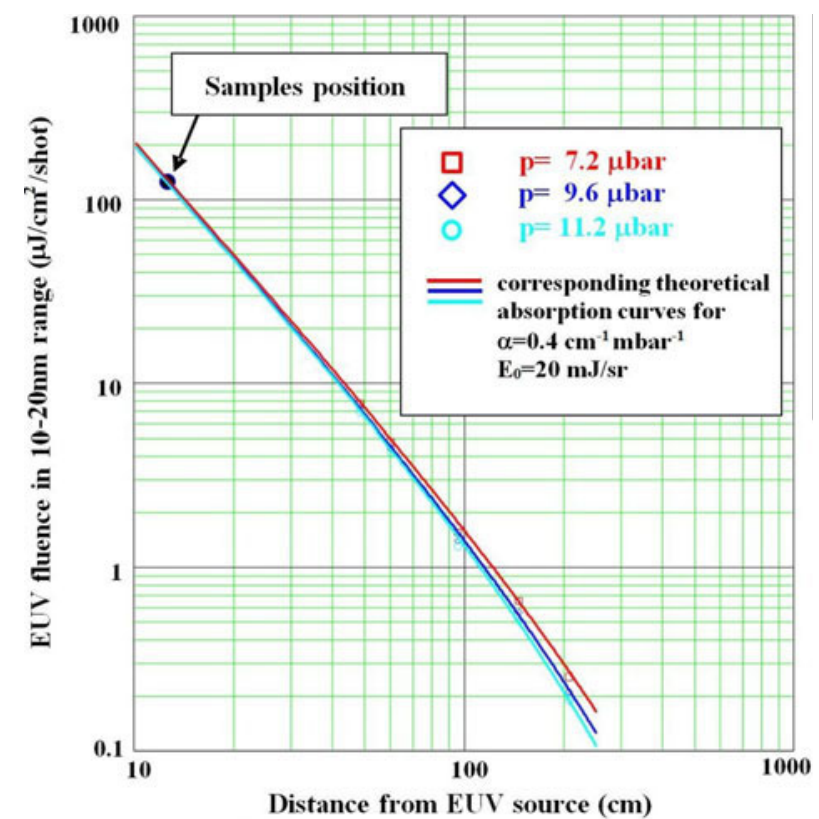

Figure 4. EUV fluence versus distance from the source for different pressure values of residual xenon: experimental data (points) and best fit with the theoretical absorption curve (solid lines, see text).

put on this direction and the EUV emission is not isotropic. It is covered by a 150 -nm-thick, Ni-mesh-supported $\mathrm{Zr}$ filter (Luxel Corp.), which is identical to the one usually put in front of the samples to reject visible and ultraviolet radiation. It was not possible, during the calibration procedure, to place the reference diode exactly in the position dedicated to the sample exposure, which is very near to the plasma source $(12.8 \mathrm{~cm})$, because of signal saturation.

In order to determine the fluence on samples during exposures, it was necessary to take into account the presence of Xe gas in the vacuum chamber during plasma source operation, due to a dynamic equilibrium between the $\mathrm{Xe}$ injection flux and the vacuum pumping speed. Accurate measurements of the reference PIN diode signal have been performed changing both the Xe pressure and the PIN diodeto-source distance. It is also crucial to take into account some spurious effects, such as a possible nonuniformity of the residual Xe gas pressure in the vacuum chamber or EUV grazing reflections on the chamber walls, which can easily generate artefacts. The Xe pressure uniformity has been confirmed by measurements taken at different points of the vacuum chamber. Undesired grazing reflections have been detected on dosimetric film and eliminated by proper screening of the PIN diode.

The measurement results are shown in Figure 4: the experimental data are very well fitted in each pressure condition by the theoretical absorption function $f(d, p)=$ $1 / d^{2} \cdot e^{-\alpha \cdot p \cdot d}$, demonstrating that spurious effects have been eliminated. The resulting spectrally averaged absorption coefficient is $\alpha=0.4 \mathrm{~cm}^{-1} \mathrm{mbar}^{-1}$, in good agreement

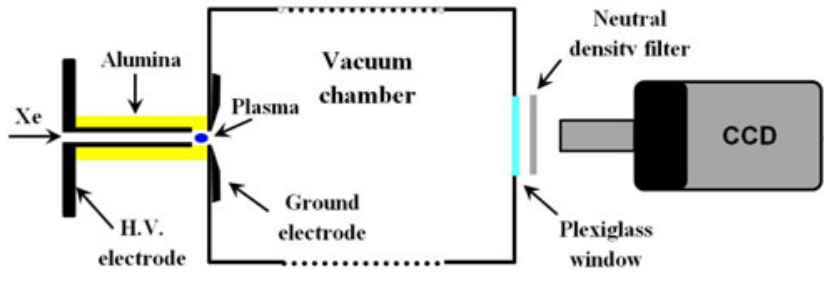

Figure 5. Schematic of the setup for recording the spatial evolution of the discharge (not in scale).

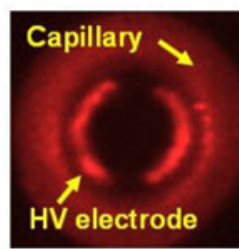

(a)

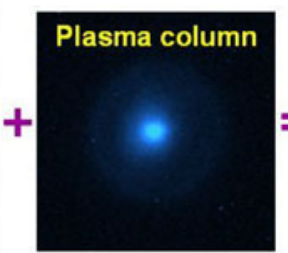

(b)

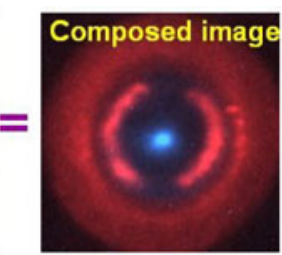

(c)
Figure 6. An example of plasma image processing: (a) picture of the HV electrode within the alumina capillary tube, converted to red; (b) picture of the visible light emitted by the plasma column, converted to blue; (c) composition of the two images. The CCD position was kept fixed throughout all the measurements.

with Henke data ${ }^{[8]}$. These curves permit one to obtain the exposure fluences on samples with an error of a few per cent.

\section{Visible time-resolved plasma spatial evolution}

The plasma spatial evolution, both in the pre-ionization and in the main discharge phases, has been observed in the visible range by a gateable CCD camera (DICAM-2, PCO Computer Optics $\mathrm{GmbH}$ ) placed at $1 \mathrm{~m}$ distance from the source. A schematic of the experimental setup is reported in Figure 5.

The camera, equipped with a telephoto lens, monitored the plasma through a Plexiglass window mounted on the vacuum chamber wall opposite to the source. The acquired plasma images have been elaborated to give them a fixed point of reference: initially, an image of the capillary tube and the HV electrode has been captured under illumination and has been superimposed on all the plasma images, which have been subsequently taken in the dark. In order to better distinguish the two contributions, they have been converted from the original grey levels to false colours - respectively, red for the structure and blue for the plasma column. An example of this image processing is shown in Figure 6.

The plasma formation during the low-current pre-ionization phase is shown in Figure 7: at the current onset, the discharge occupies only a small portion of the capillary and only after about $2 \mu$ s does it fill the whole tube. Hence, the main discharge triggering delay has been set slightly longer 

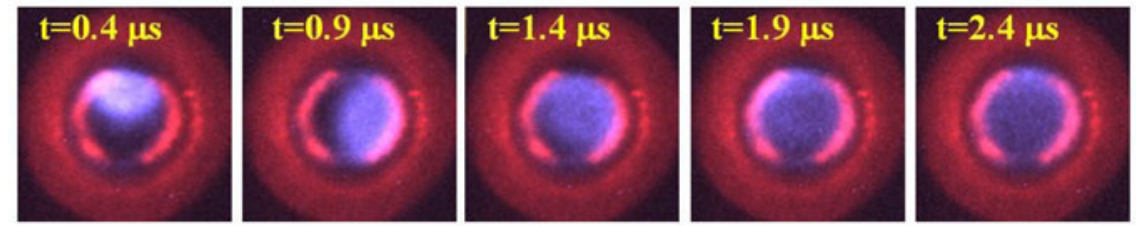

Figure 7. Sequence of false colour images of the discharge during the pre-ionization phase. The exposure time is $0.1 \mu$ s, on distinct shots. The indicated time $t$ on each image is the delay with respect to the current onset.
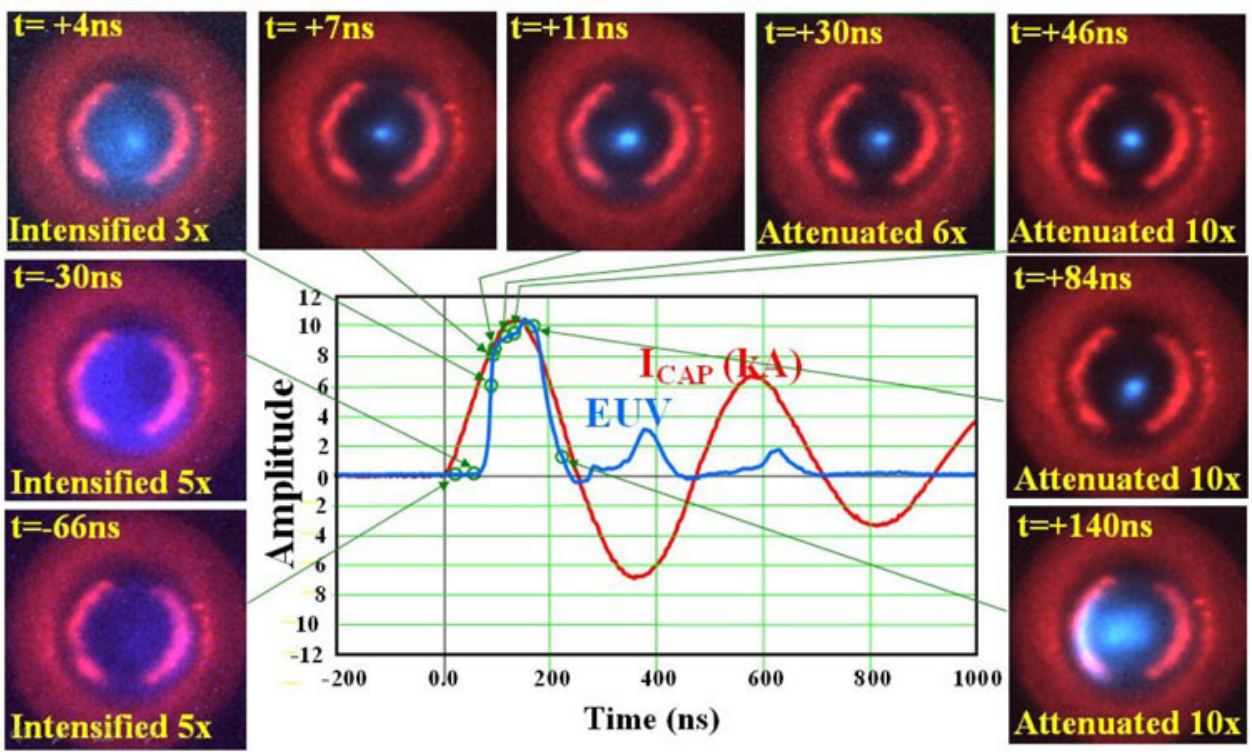

Attenuated 10x

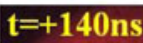

Figure 8. False colour images of the visible light emission from the plasma during the main discharge. Gate time was $20 \mathrm{~ns}$. The indicated time $t$ on each image refers to the acquisition delay from EUV rise (50\% of maximum). The temporal position of each image with respect to the EUV power emission (blue curve) is also indicated by green circles in the graph. The red curve is the discharge current $I_{\text {CAP. }}$.

than the pre-ionization developing time. As the images shown in Figure 7 are acquired on successive shots, it is evident that the onset of the pre-ionization discharge occurs at a random point of the capillary internal surface.

The evolution of the main discharge was observed using a gate time of $20 \mathrm{~ns}$ for each acquisition. Observing the sequence reported in Figure 8, the pinch effect is clearly visible: after the collapse, the plasma-column diameter is reduced by more than one order of magnitude with respect to its starting value, reaching a minimum size below $300 \mu \mathrm{m}$. Since the reported images again refer to distinct shots, it is evident that the plasma shot-to-shot position stability is very good.

The sequence of pictures also shows that the plasma collapse and its successive relaxation temporally coincide with the EUV emission rise and fall, respectively, as expected. It is worth noticing that the visible emission power grows both during and after the EUV main pulse, so that the optical density of a neutral filter placed in front of the camera had to be increased for longer delays in order to avoid camera saturation (see Figure 5). This could be related to the fact that the xenon low-charge-state ions population, i.e., those responsible for visible light emission, grows continuously during the main discharge. In contrast, if the plasma evolution followed a sequence of corona equilibrium states ${ }^{[10]}$, the visible light emission, normalized to the electrical input power, should decrease in correspondence to the EUV emission, when the plasma temperature $T$ is maximized. In fact, in the corona equilibrium case, the average ion charge state $Z$ would depend only on $T$, scaling approximately as: $Z \propto T^{0.4}$ (Ref. [11]). Moreover, the population of weakly ionized atoms (Xe II and Xe III ions) would be maximized just for low plasma temperatures $(T \sim 20000 \mathrm{~K})$ (see Ref. [12] and Figure 34 in Ref. [7]). Plasma temperatures necessary for EUV emission $(T \sim$ $400000 \mathrm{~K}$ ) imply $Z$ high enough to strongly reduce the visible light emission. Since a quite large $Z$ is needed to emit EUV radiation ${ }^{[13]}$, in our conditions the xenon plasma cannot reach an equilibrium state during the main discharge, due to the too short time of evolution. Therefore, in this non-equilibrium condition, the low- $Z$ population can grow continuously with time. 


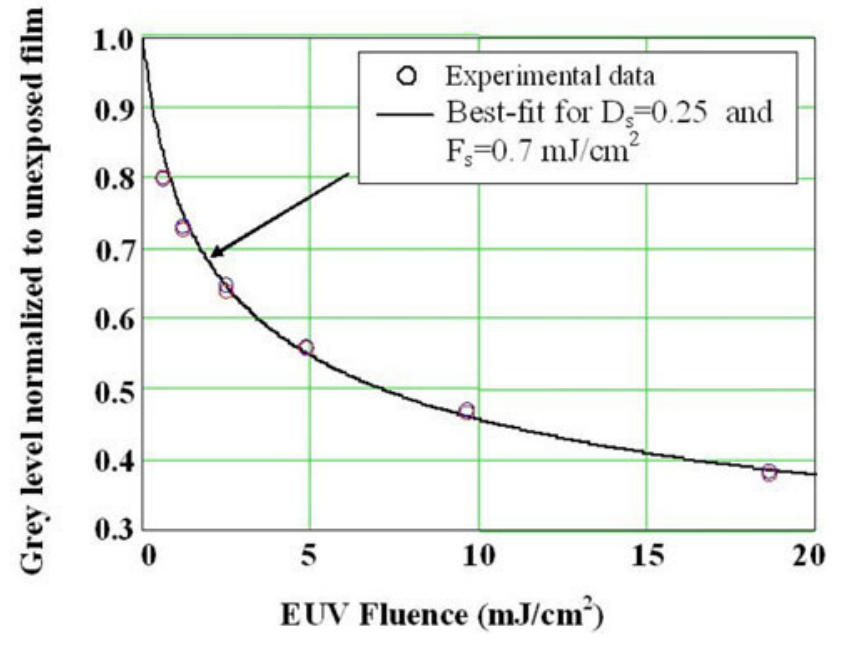

Figure 9. Calibration curve of the Gafchromic HD-V2 dosimetric film in the 10-20 nm spectral range: experimental grey levels (acquired by a quadraticsensitivity 16-bit scanner, Canon, CanoScan 8400F, red channel) versus EUV fluence (circles) and best fit with the photographic thick-film function (see text).

\section{Calibrated EUV imaging by dosimetric film}

Gafchromic HD-V2 dosimetric film is another powerful tool that can be used to characterize EUV plasma sources. The exposure to ionizing radiation modifies its optical density in a reproducible way depending on the impinging fluence, allowing its use as a quantitative imaging detector. Since the calibration curve provided by the company applies to hard $\mathrm{X}$-rays, the film has been exposed to determined fluences in the $\lambda=10-20 \mathrm{~nm}$ EUV region by using the DPP source. It is expected that in this spectral range the film optical density versus fluence follows the photographic thick-film formula, due to the above-mentioned EUV short penetration length:

$$
D=D_{s} \cdot \ln \left(1+\frac{F}{F_{S}}\right)
$$

where $D$ is the film optical density normalized to the unexposed film, $D_{s}$ is the saturation optical density, $F$ is the EUV fluence and $F_{s}$ is the EUV saturation fluence. Since the film manufacturer suggests using the red component of the RGB images thanks to its larger response, all the reported grey levels correspond to that channel.

In Figure 9 the experimental film response, expressed as the grey level of the scanned film, is reported and fitted by Equation (1), taking into account that the grey level $g$ of our 16-bit scanner is proportional to the root square of the light intensity:

$$
\mathrm{g}=10^{\left[D_{s} / 2 \cdot \ln \left(1+F / F_{s}\right)\right]} .
$$

The very good agreement between the experimental data and the best-fit curve allows quantitative use of the dosimetric film also in the EUV region.

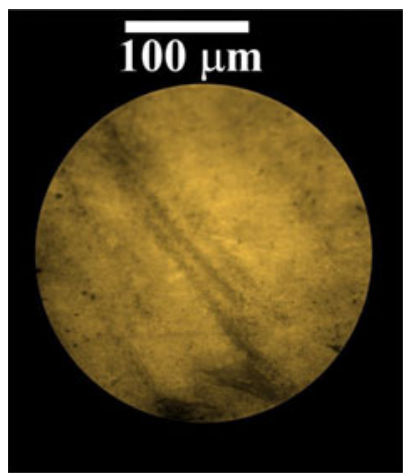

Figure 10. Portion of a radiography of an $\mathrm{Al}$ filter. A LiF crystal, covered by a $0.8-\mu \mathrm{m}$-thick $\mathrm{Al}$ membrane, has been exposed to 8000 shots at $10 \mathrm{~cm}$ from the DPP source and observed by using a fluorescence microscope at $50 \times$ magnification. The filter irregularities are visible as luminescent patterns.

In particular, by placing the film at $12.8 \mathrm{~cm}$ from the source (sample exposure position) and at $96 \mathrm{~cm}$ from the source (one of the experimental points of Figure 4), it has been possible to verify that the two fluence values, determined from the film grey levels, fit the theoretical curves of Figure 4 well.

\section{EUV plasma source applications}

Thanks to the extensive characterization of the EUV radiation produced by the DPP source, several applications have benefited from its controlled operation. In Figure 10 we present a small portion of a $5 \times 5(\mathrm{~mm})$ aluminium filter radiographically imaged on a lithium fluoride $(\mathrm{LiF})$ crystal, where EUV radiation generates stable colour centres ${ }^{[14]}$ which can be observed by using a fluorescence microscope. The sample, placed in the vacuum chamber at $10 \mathrm{~cm}$ from the plasma, has been exposed to 8000 shots of the DPP source, corresponding to a total emitted fluence of $2 \mathrm{~J} / \mathrm{cm}^{2}$. In this case the effective released dose to the crystal was much lower, $\simeq 0.1 \mathrm{~J} / \mathrm{cm}^{2}$, because most of the EUV radiation is emitted by the DPP source at $\lambda<17 \mathrm{~nm}^{[6,7]}$, while the $\mathrm{Al}$ filter mostly transmits EUV radiation for $\lambda>17 \mathrm{~nm}^{[8]}$.

Also EUV direct exposures of novel photoresists have been carried out in the framework of a project, funded by the CARIPLO Foundation, which involves ENEA and the Universities of Pavia and Padova. An example of EUV exposure of a resist synthesized at Padova University is shown in Figure 11.

Finally, the controlled irradiation of $\mathrm{LiF}$ thin films, evaporated at the MNF Laboratory of ENEA, allowed the creation of anticounterfeiting tags by lithographic writing techniques $^{[15]}$.

\section{Diagnostics relevance for LPPs}

As mentioned in the Introduction, the development of EUVemitting plasmas is of paramount interest in the field of 


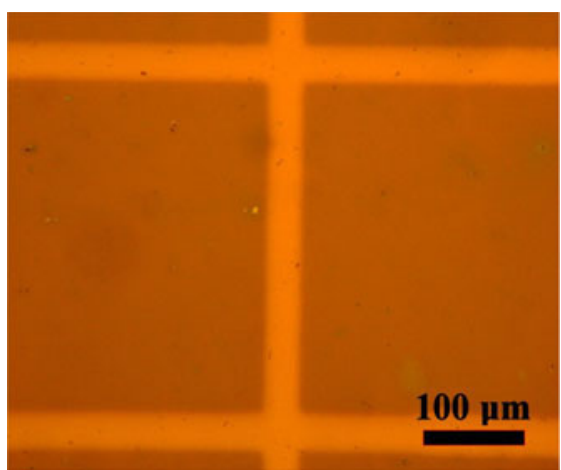

Figure 11. EUV exposure of an innovative zirconia-loaded photoresist behind a $150-\mathrm{nm}$ thick $\mathrm{Zr}$ filter: the Ni mesh supporting the filter is imaged on the resist. The grid period is $363 \mu \mathrm{m}$. The exposure total fluence was $\approx 600 \mathrm{~mJ} / \mathrm{cm}^{2}$ released in 7000 shots, at $128 \mathrm{~mm}$ from the source. The wire edges are blurred due to a distance of approximately $2 \mathrm{~mm}$ between the filter and the photoresist.

next-generation lithography, the choice between LPP and DPP sources still being an open question. As a matter of fact, several critical issues have yet to be overcome and a deeper understanding of the plasma characteristics is needed.

In spite of the evident differences in plasma heating methods between LPP and DPP, many plasma parameters are similar, with regard to temperatures, materials, residual gases in the vacuum chambers, and surrounding environment. The major differences between LPP and DPP are related to the geometry for the collection of the EUV emitted radiation and for the mitigation of the produced debris ${ }^{[1]}$.

For these reasons, the diagnostics described in this paper are directly applicable to laser-generated plasmas for their temporal and spatial characterization. It is also worth mentioning the particular case of X-ray lasers, both those operating as capillary discharges and those relying on collisional methods, which can particularly benefit, for example, from fast-gated image acquisition, especially for synchronization purposes when used as amplifying media ${ }^{[16]}$.

\section{Concluding remarks}

An EUV source, based on a DPP, is operating at the ENEA Frascati Laboratories. Several diagnostics have been exploited to characterize the EUV plasma source: absolute PIN diodes, a gateable CCD camera in the visible range, and a dosimetric film which has been also calibrated in the 10-20 nm wavelength interval. The described diagnostics can be applied also to other EUV plasma sources, such as LPPs. Just such an LPP EUV/soft X-ray source is also operating at the ENEA Frascati Laboratories ${ }^{[17]}$. Analogous diagnostics have been applied for its characterization, aimed at a strict control of the spatial, spectral and energy features of the emitted radiation, which is particularly essential for its application to EUV microlithography ${ }^{[18]}$. Such assessed sources allow controlled and reproducible direct EUV exposures for a variety of applications.

\section{Acknowledgements}

The presented research was partially funded by the Italian Ministry for University and Research (FIRB project no. RBNE01ABPB) and by the Cariplo Foundation (project no. 2012-0816).

\section{References}

1. V. Y. Banine, K. N. Koshelev, and G. H. P. M. Swinkels, J. Phys. D Appl. Phys. 44, 253001 (2011).

2. V. M. Borisov, Quantum Electron. 44, 1077 (2014).

3. J. C. Valenzuela, J. Phys. Conf. Ser. 511, 012023 (2014).

4. K. Bergmann, G. Schriever, O. Rosier, M. Muller, W. Neff, and R. Lebert, Appl. Opt. 38, 5413 (1999).

5. B. Huang, Y. Takimoto, M. Watanabe, and E. Hotta, Japan. J. Appl. Phys. 50, 06GB09 (2011).

6. P. Zuppella, A. Reale, A. Ritucci, P. Tucceri, S. Prezioso, F. Flora, L. Mezi, and P. Dunne, Plasma Sources Sci. Technol. 18, 025014 (2009).

7. L. Mezi and F. Flora, ENEA Technical Report RT/2012/15/ENEA, http://opac.bologna.enea.it:8991/RT/201 2/2012_15_ENEA.pdf (September 2012 in Italian).

8. B. L. Henke, E. M. Gullikson, and J. C. Davis, At. Data Nucl. Data Tables 54, 181 (1993) http://henke.lbl.gov/optical_const ants.

9. For an extensive technical description, see the web site: http://optodiode.com/pdf-library/1.\%20IRD\%20-Photodiodes $\% 20-\% 20$ AXUV\%20Operating\%20Principles\%20and $\% 20 \mathrm{~A}$ pps.pdf.

10. R. J. Goldston and P. H. Rutherford, Introduction to Plasma Physics (Institute of Physics, Bristol and Philadelphia, 1995).

11. M. Masnavi, N. Nakajima, and K. Horioka, J. Plasma Fusion Res. 79, 1188 (2003).

12. Y. Kobayashi and R. M. Neren, J. Quant. Spectrosc. Radiat. Transfer 12, 1647 (1972).

13. E. B. Saloman, J. Phys. Chem. Ref. Data 33, 765 (2004).

14. G. Baldacchini, S. Bollanti, F. Bonfigli, F. Flora, P. Di Lazzaro, A. Lai, T. Marolo, R. M. Montereali, D. Murra, A. Faenov, T. Pikuz, E. Nichelatti, G. Tomassetti, A. Reale, L. Reale, A. Ritucci, T. Limongi, L. Palladino, M. Francucci, S. Martellucci, and G. Petrocelli, Rev. Sci. Instrum. 76, 113104 (2005).

15. P. Di Lazzaro, S. Bollanti, F. Flora, L. Mezi, D. Murra, A. Torre, F. Bonfigli, R. M. Montereali, and M. A. Vincenti, Proc. SPIE 8677, 86770T (2013) .

16. S. Suckewer and P. Jaeglé, Laser Phys. Lett. 6, 411 (2009).

17. S. Bollanti, F. Bonfigli, E. Burattini, P. Di Lazzaro, F. Flora, A. Grilli, T. Letardi, N. Lisi, A. Marinai, L. Mezi, D. Murra, and C. Zheng, Appl. Phys. B 76, 277 (2003).

18. S. Bollanti, P. Di Lazzaro, F. Flora, L. Mezi, D. Murra, and A. Torre, Eur. Phys. Lett. 84, 58003 (2008). 